\title{
A METHOD ON MEASUREMENTS OF SOLAR VELOCITY FIELDS
}

\author{
XIAO-MA GU, JUN LIN, KE-JUN LI
}

Yunnan Observatory, P.O.Box 110, Kunming, YN 650011, P.R. China

\begin{abstract}
A new method of 'multi-cloud model'(MCM) is proposed and two application examples are given in the paper.
\end{abstract}

A spectral line profile of solar active object(SAO) contains a great deal of imformation about the dynamical characteristics, physical properties and structures of the SAO, So, how to extract much information of the SAO from the spectral data obtained has been one of the very important subjects.

'Cloud model'(CM) method was proposed (Beckers,1964), it is assumed that source function $\mathrm{S}$ is constant in $H_{\alpha}$ line and four parameters ( $\mathrm{S}$, optical depth at the line center $\tau_{0}$,Doppler width of the line $\Delta \lambda_{D}$, and the line-of-sight velocity V) do not vary in a single cloud, and the background is homogeneous, the profile is Gaussian and only broadened by Doppler effect. Thus, the four physical parameters are derived. The method was applied to Balmer's lines (GrossmannDoerth and Von Uexküll 1971,1977, Hanaoka et al.1989 and Alissandrakis et al.1990).

Mein and Mein (1988) improved Beckers's method and proposed 'differential cloud models'(DCMs). The method allows an inhomogeneous background and the existences of gradient and shearing of the velocity inside the 'cloud', thus, the applicable range of the CM method was expanded. The method was applied to the studies of post-flare loops by Schmieder et al.(1988), Heinzel et al.(1992) and Gu et al.(1992). Unfortunately, all of these methods are only suitable for symmetric profiles.

A number of observations show that the profiles in flares, eruptive prominences, material ejections etc. are usually asymmetric (non-Gaussian). Luo et al.(1987) proposed 'two-layer model'(TLM) and derived the fields of some physical quantities for the flare of May 16,1981. No good general method has been found to process well those non-Gaussian profiles up to now except for the TLM. Now, we try to extend the CM to 'multi-cloud model'(MCM) so as to process general asymmetric profiles of SAO.

We consider that a non-Gaussian profile is the result of combination of $m$ Gaussian sub-profiles which are produced by $m$ emitting origins (so-called $m$ 'clouds' ) along the line-of-sight. $S$ and $V$ in each 'cloud' are assumed to be constants. Thus, the observed intensity emitted by the $m$ 'clouds' $I_{\lambda}^{\text {obs }}$ will be: 


$$
I_{\lambda}^{o b s}=I_{0, \lambda} e^{-\tau_{\lambda}}+\sum_{j=1}^{m} S_{j}\left(1-e^{-\tau_{\lambda, j}}\right) \exp \left(-\sum_{i=0}^{j-1} \tau_{\lambda, i}\right),
$$

where we let $\tau_{\lambda, 0}=0, I_{0, \lambda}$ is the background intensity, $\tau_{\lambda}=\sum_{j=1}^{m} \tau_{\lambda, j}$ is the total optical depth of the $m$ 'clouds' and

$$
\tau_{\lambda, j}=\tau_{0, j} \exp \left[-\left(\frac{\lambda-\lambda_{0}-\Delta \lambda_{0, j}}{\Delta \lambda_{D, j}}\right)^{2}\right]
$$

where $\Delta \lambda_{0, j}=\lambda_{0} V_{j} / C, S_{j}, \tau_{0, j}, \Delta \lambda_{D, j}$ and $V_{j}$ are the corresponding quantities of the $j^{\text {th }}$ cloud. Then, the contrast profile $C(\lambda)=\frac{I_{0}^{000}-I_{0, \lambda}}{I_{0, \lambda}}$ is as follows:

$$
C(\lambda)=e^{-\tau_{\lambda}}+\frac{1}{I_{0, \lambda}} \sum_{j=1}^{m} S_{j}\left(1-e^{-\tau_{\lambda, j}}\right) \exp \left(-\sum_{i=0}^{j-1} \tau_{\lambda, i}\right)-1,
$$

Based on principle of the least square method, we can fit the theoretical profiles $C(\lambda)$ given by Eq.(3) into the observational ones and the four physical parameters in each 'cloud' may be derived under conditions of certain approximation and precision.

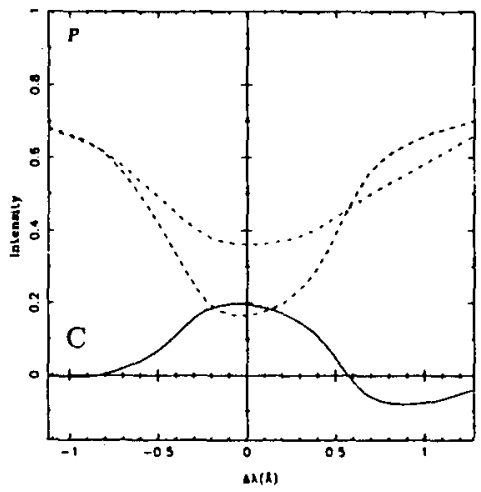

Figure 1: Ho profile ( $P$ ) and coutrast profile (C) in a post-flare loop observed with MSDP ots May 5.1989 at Meition Observatory. The olserved coutrast profile is in solid line, the fitting one in dotted liue. The values of the derived physical parameters are listed in Table I.

\begin{tabular}{|c|c|c|c|c|c|c|}
\hline $\begin{array}{l}\text { Ohingrved } \\
\text { dele }\end{array}$ & $\begin{array}{l}\text { Choud } \\
\text { number }\end{array}$ & Dbfects & (I I eone) & $\overline{0}$ & $\begin{array}{l}\Delta \lambda_{D} \\
(\lambda)\end{array}$ & $\begin{array}{l}\text { V } \\
\text { (lens/s) }\end{array}$ \\
\hline May $\mathbf{5 . 1 5 0 0}$ & $m=2$ & $\begin{array}{l}\text { pnot-inere } \\
\text { inop }\end{array}$ & 0.44 & 0.50 & 0.55 & 378 \\
\hline \multirow{3}{*}{ Aus.17.1800 } & & flare ribbon & 0.50 & 0.04 & 0.73 & -8.8 \\
\hline & $n=3$ & boop 1 & 0.51 & 0.53 & 0.36 & -53.90 \\
\hline & & $\begin{array}{l}\text { loop } 2 \\
\text { loop } 3\end{array}$ & $\begin{array}{l}0.62 \\
0.93\end{array}$ & $\begin{array}{l}0.38 \\
0.18\end{array}$ & $\begin{array}{l}0.30 \\
0.21 \\
\end{array}$ & $\begin{array}{l}-18.45 \\
10.18\end{array}$ \\
\hline
\end{tabular}

TABLE I: The phyical parameters of SAOs derived from the MCM method.

TABLE II: A comparion of the resalts obtrined wib s

\begin{tabular}{|c|c|c|c|c|c|}
\hline Paranueters & $\bar{i}$ & $\overline{2}$ & $\mathbf{3}$ & 1 & $\overline{5}$ \\
\hline$\xi_{1}\left(l_{\operatorname{com} t}\right)$ & $\overline{0.437}$ & $\overline{0.437}$ & $\overline{0} .371+3$ & 0.512 & 0.515. \\
\hline$s_{2}\left(I_{\text {ems }}\right)$ & 0.199 & 0.198 & 0.200 & 0.477 & $0.5 \%$, \\
\hline$s_{1}\left(t_{\text {cont }}\right)$ & $0.51+2$ & $0,498 \cdot 2$ & $0.1+1+3$ & 0.970 & $0.0^{\circ}$ \\
\hline$\pi_{0.1}$ & 0.1000 & 0.681 & $0.65 t-2$ & 0.530 & 0.530 \\
\hline 0. & 1.340 & 1.340 & 0.868 & 0.375 & v.37s \\
\hline T. & $0.191-2$ & $0.290-1$ & $0.930-3$ & 0.150 & 0.130 \\
\hline$\Delta \lambda_{D, 1}(\lambda)$ & 0.378 & 0.376 & 0.426 & 0.378 & 0.380 \\
\hline$\Delta \lambda_{D,},(\lambda)$ & 0.206 & 0.206 & 0.197 & 0.236 & 0.290 \\
\hline$\Delta \lambda_{D .}(A)$ & 0.393 & 0.393 & 0.143 & 0.319 & 0.210 \\
\hline$V_{1}(\mathrm{k} / \mathrm{m} / \mathrm{o})$ & .52 .74 & -52.74 & -52.91 & -83.99 &.$\$ 3.99$ \\
\hline $13(k \mathrm{~m} / \mathrm{t})$ & -17.48 & -17.49 & -18.99 & -18.20 & -18.45 \\
\hline $1,1 \mathrm{~km} / \mathrm{e})$ & 7.95 & 7.95 & 6.85 & 11.60 & 10.18 \\
\hline$S_{1} \times 0.1$ & 0.288 & 0.289 & 0.211 & $0.8 \mathrm{il}$ & 0.271 \\
\hline$S_{2} \times 0.2$ & 0.237 & 0.267 & 0.17 & 0.179 & 0.100 \\
\hline$s_{2} \times \pi_{0,3}$ & 0.136 & 0.136 & 0.134 & 0.139 & 0.139 \\
\hline
\end{tabular}
different iuitial Parameters for the Loop syotesu of Aug. 17,1989 at Yunnan Obs..

we apply the MCM method to process spectral data of a two-ribbons flare observed on the disk with the multi-channel double pass spectrograph (MSDP, Mein et al.1977) on May 5,1989 at Meudon Observatory. A detail description of the flare is given by Gu et al.(1992). At some spatial sites, the contrast profile of $H_{\alpha}$ line observed consists of both negative and positive (cf. Fig.1), the former corresponds to the post-flare loop ('cloud' 1) and the latter to the flare ribbon ('cloud' 2). As a convenient first approximation, we take the $S$ in the flare ribbons as a constant. Then, we fitted $H_{\alpha}$ contrast profile given by Eq.(3) (let $\left.m=2\right)$ 
to the observational one, deducing eight physical parameters which are listed in Table I. The profile computed is also given in Fig.1.

We applied the MCM method to process the spectral data of a flare loop system observed on solar limb with the 2-D multi-band spectrograph (Xuan and Lin 1992) at Yunnan Observatory on August 17, 1989. A preliminary report of the flare loop system was given by Xuan et al.(1990). The $H_{\alpha}$ photograph and the $H_{\beta}$ spectral picture are given in Figures 2a and 2b. The corresponding intensity profile of $H_{\beta}$ line observed is shown in Figure 3. One can find from the Figure 2a that on the slit sites there are some loops overlapped one another along the line-of-sight, thus the profile shows multi-peak shape owing to the different velocities of the loops (Figure 3). It is impossible to analyse and process such kind of profile with traditional method. However, the MCM method allows it possible to derive some physical parameters within the loops by using Eqs.(1)(3) (where $m=3$ and the $I_{0, \lambda}=0$ in Eq.(1)) to fit the profile shown in Fig.3. The theoretical intensity profile is also given in Fig.3. The physical parameters derived for the loop system are listed in Table I. One can find from Figs.1 and 3 that the intensity profiles computed are all in good agreement with that observed except for the line wings, it shows a good accuracy in the method.

In the computation, it is necessary to select suitable initial values of these parameters. For $m=3$, many trial calculations show that the parameters derived show some differences for different initial values of the parameters, but within a certain precision, the $V$ and $\Delta \lambda_{D}$ derived are almost same, the five computed results are given in Table II.. Although $S$ and $\tau_{0}$ vary, the product of $S \times \tau_{0}$ is almost constant for a fixed 'cloud'(c.f. Table II), it shows that the 'cloud' is optically thin and additional conditions are requested to separate $S$ and $\tau_{0}$. If 'cloud' is optically thick (saturation), then observation only reflects the value of $S$ in the 'cloud'. If the $S$ in a 'cloud' (such as in flares) varies with depth, we may divide it into $m$ 'sub-clouds', then MCM method may be applied.

In order to choice well a set of the initial values of parameters and get the solution of Eq.(3), it is important to identify a corresponding relation between 'clouds' and the peaks of a profile at some spatial sites, it is easy to do it if the 'clouds' show different properties, but it is difficult for those 'clouds' that are identical in physical properties. Generally, we can do it and select the initial parameters by continuity of each 'cloud'(loop) near some sites(Gu et al.1992). Of course, other much information on the 'clouds' will be required.

In principle, any profile can be fitted with ' $m$-cloud model' if $m$ is large enough(Mein,1992). But the larger is $m$, the more complex is the mathematical computation and the lower is the accuracy of the derived parameters. In fact, $m$ will not too big due to the 'cloud' absorptions, the radiation observed mainly comes from the first 'cloud'(closest to the observer) then from the second one, a few of them comes from the third one (for $H_{\alpha}, H_{\beta}$ lines). None profile with more than 3 peaks has been found after searching all profiles of the loop system of Aug. 17, 1989. For $m=2$ and 3, the solution may not be unique unless other physical reasons can account for additional assumptions (Mein,1992). However, for a given precision, a set of reasonable solution is able to be obtained if the initial parameters are well chosen (Gu et al.1992). On the other hand, making use of a suitable compute code, we can reject unreasonable solutions and retain the reasonable one. A descenting dimention computation method has been proposed 
by $\mathrm{Li}(1992)$, the application of the method will be given in the future.

\section{ACKNOWLEDGEMENTS}

The authors would like to thank Profs. B. Schmieder and P. Mein for providing the MSDP data and helpful discussions. We will also thank the staff of VAX-8350 Computer at Yunnan Observatory for their helps.
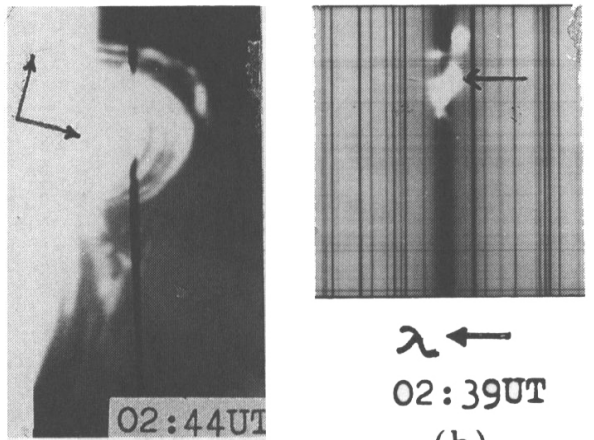

$02: 390 T$

(b)

Figure 2: (a) Ha filtergram of post-flare loop system observed on Ang.17.1989 with 2$D$ nulti-band spectrograph at Ynnnan Obs. Blach live refers to the position of incident slit. (b) Spectrogran of $\mathrm{B} \beta$ line of the same object. Arrow points to the site where the profile slown in Fig. 3 was constructed.

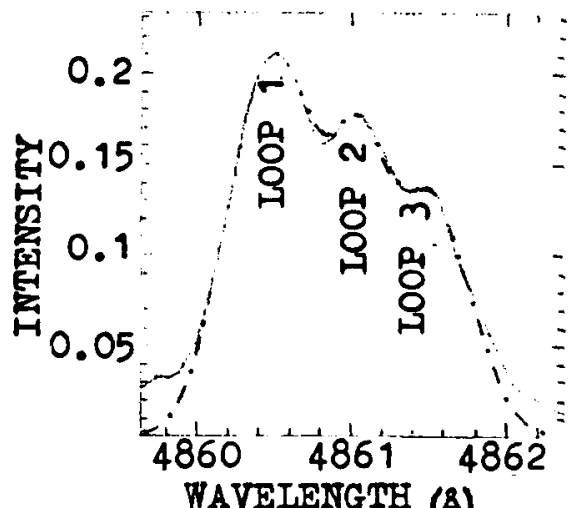

Figure 3: Three-peak $\mathrm{B} 3$ profile (in solid line) of post-flare loop system observed at the sular liwh on Aug. 1\%, 1989 at Yuunan Obsertatory. The dashed and dotted line refers to the fitting profile calculated with Eq.(3) theoretically. The values of the derived physical parameters are listed in Table I.

\section{REFERENCES}

Alissandrakis,C.E.,Tsiropoula,G.,Mein,P.:1990, A \& A , 230,200.

Beckers,J.M.:1964,Ph.D.Thesis,Utrecht; AFCRL Env.Res.Papers, N0. 49.

Grossmann-Doerth,U. and von Uexküll,M.:1971, Solar Phys., 20,31.

Grossmann-Doerth,U. and von Uexk üll,M.:1977, Solar Phys., 55,321.

Gu,X.M.,Lin,J.,Luan,T.,Schmieder,B.:1992, A \& A , 259,649.

Hanaoka,Y., Kurokawa,H.:1989, Solar Phys., 124,227.

Heinzel,P.,Schmieder,B., Mein,P.:1992, Solar Phys., 139,81.

Luo,Z.:1987, MSc Thesis, Yunnan Observatory.

Li,K.J.: 1992, Pub. of Yunnan Obs., in press.

Mein,P. and Mein,N.:1988, A \& A , 203,162.

Mein,P.: 1977, Solar Phys., 54,45.

Mein,P.:1992, private communication.

Schmieder,B.,Mein,P.,Malherbe,J.,Forbes,T.,:1988,Adv. Space Res.,8, No.11,145.

Xuan,J.Y. and Lin,J.:1992, Solar Phys., in press.

Xuan,J.Y.,Luo,Z.,Ye,H.L.,Zhong,S.H.,:1990, Pub. of Yunnan Obs., 4, 181. 